\title{
Semantic interference in picture naming during dual-task performance does not vary with reading ability
}

\author{
Vitória Piai (D), Ardi Roelofs and Ingeborg Roete \\ Radboud University Nijmegen, Donders Institute for Brain, Cognition and Behaviour, Centre for Cognition, \\ Nijmegen, the Netherlands
}

(Received 11 July 2014; accepted 19 October 2014; first published online 16 December 2014)

\begin{abstract}
Previous dual-task studies examining the locus of semantic interference of distractor words in picture naming have obtained diverging results. In these studies, participants manually responded to tones and named pictures while ignoring distractor words (picture-word interference, $\mathrm{PWI}$ ) with varying stimulus onset asynchrony (SOA) between tone and PWI stimulus. Whereas some studies observed no semantic interference at short SOAs, other studies observed effects of similar magnitude at short and long SOAs. The absence of semantic interference in some studies may perhaps be due to better reading skill of participants in these than in the other studies. According to such a reading-ability account, participants' reading skill should be predictive of the magnitude of their interference effect at short SOAs. To test this account, we conducted a dual-task study with tone discrimination and PWI tasks and measured participants' reading ability. The semantic interference effect was of similar magnitude at both short and long SOAs. Participants' reading ability was predictive of their naming speed but not of their semantic interference effect, contrary to the reading ability account. We conclude that the magnitude of semantic interference in picture naming during dual-task performance does not depend on reading skill.
\end{abstract}

Keywords: Dual-task performance; Picture-word interference; Response-selection bottleneck; Reading ability; Semantic interference

Lexical selection in spoken word production is a hotly debated topic in the language production literature (e.g., Dell'Acqua, Job, Peressotti, \& Pascali, 2007; Janssen, Schirm, Mahon, \& Caramazza, 2008; Kleinman, 2013; Piai \& Roelofs, 2013; Piai, Roelofs, \& Schriefers, 2011; Schnur \& Martin, 2012). Several investigators maintain that lexical selection in word production is a competitive process (Levelt, Roelofs, \& Meyer, 1999; Roelofs, 1992; Starreveld \& La Heij, 1996). Behavioural evidence for competitive selection in word production comes, for example, from studies of picture naming in which the amount of lexical competition is manipulated by simultaneously presenting distractor words, the so-called picture-word interference (PWI) paradigm (Rosinski, 1977; for reviews, see Abdel Rahman \& Melinger, 2009; Glaser, 1992; Roelofs, 2007). A common finding in PWI studies is that picture-naming response time (RT) is longer in the related (e.g., a picture of a car combined with the distractor word bus) than in the unrelated (pictured car, word pen) condition, an effect referred to as semantic interference (e.g., Levelt et al., 1999; Roelofs, 1992; Schriefers, Meyer, \& Levelt, 1990).

Correspondence should be addressed to Vitória Piai, University of California, 132 Barker Hall, Berkeley, CA 94720-3190, USA. E-mail: v.piai.research@gmail.com

This work was supported by the Netherlands Organization for Scientific Research [grant number 400-09-138]. 
According to the competition view, semantically related distractor words are primed by the picture (La Heij, Dirkx, \& Kramer, 1990) and are therefore stronger competitors to the picture name than unrelated distractor words. This enhanced competition in the related condition prolongs the duration of word selection relative to the unrelated condition (Roelofs, 1992), explaining the semantic interference effect in the RTs. Thus, according to the theory of competitive lexical selection, the semantic interference effect arises at the stage of lexical response selection.

Recently, a dual-task procedure, known as the psychological refractory period (PRP) paradigm (Pashler, 1994), has been used to test the assumption that the semantic interference effect arises during lexical selection (Dell'Acqua et al., 2007; Kleinman, 2013; Piai, Roelofs, \& Schriefers, 2014; Schnur \& Martin, 2012). With this procedure, participants have to respond quickly and accurately to two stimuli (S1 and S2) with the requirement that a response to $\mathrm{S} 1$ is given before a response to $\mathrm{S} 2$. The stimulus onset asynchrony (SOA) between S1 and S2 is usually varied to determine the extent to which certain processes are delayed due to concurrent processing. In previous studies examining the locus of the semantic interference effect, a manual tone discrimination task was used as Task 1, followed by a PWI task (Task 2) with semantically related and unrelated picture-word pairs.

In the context of overlapping tasks, response selection is predominantly viewed as a processing bottleneck (Pashler, 1994)—that is, only one response can be selected at a time. Thus, under the PRP procedure, selecting a response for Task 2 has to wait until a response for Task 1 has been selected, as illustrated in Figure 1. If the SOA between S1 and S2 is long (Figure 1a), then the response-selection stages of the two tasks do not overlap, and effects of a Task 2 manipulation (e.g., the semantic interference effect) are usually observed. If the SOA between S1 and S2 is short, effects of a Task 2 manipulation may be present (Figure 1b) or absent (Figure 1c). If an effect is present at short SOAs, it is assumed that the effect in question emerges at response-selection or postselection stages (Figure 1b). If an effect is (a)

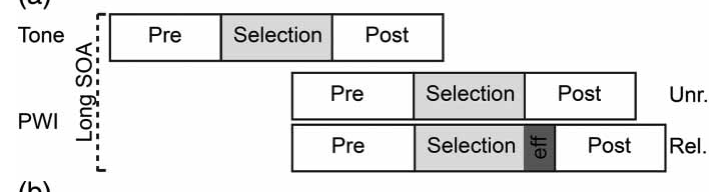

(b)

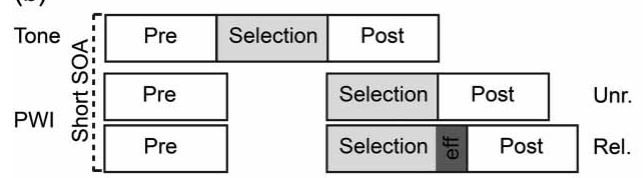

(c)

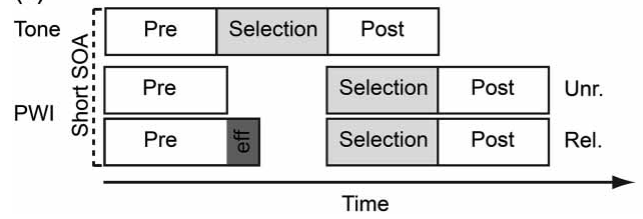

Figure 1. Schematic illustration of a response-selection bottleneck account of effects in the psychological refractory period procedure. $E f f=$ effect; $S O A=$ stimulus onset asynchrony; $P W I=$ pictureword interference; unr. = unrelated; rel. = related. (a) At long $S O A s$, the semantic interference in Task 2 is observed in the response time (RT). (b) At short SOAs, the semantic interference effect in Task 2 is observed in the RTs if it arises during response selection or later stages. (c) At short SOAs, the semantic interference effect in Task 2 is absent in the RTs if it arises during preselection stages.

absent, it is assumed that the effect is absorbed into the period in which Task 2 processes have to wait for the response-selection stage in Task 1 to be completed, a waiting period known as cognitive slack (Pashler, 1994). This absorption is taken as evidence that the effect in question emerges prior to response selection (Figure 1c).

In previous studies examining the locus of the semantic interference effect, using varying SOAs of $0,100,350,800$, and $1000 \mathrm{~ms}$, two main patterns have been observed (for an overview, see Piai et al., 2014). In some studies, the semantic interference effect was absent (or much reduced) at short SOAs, but present at long SOAs (Ayora et al., 2011; Dell'Acqua et al., 2007; Kleinman, 2013; van Maanen, van Rijn, \& Taatgen, 2012). That is, the effects of SOA and distractor type were underadditive, suggesting that the locus of the semantic interference effect is at preselection stages, as shown in Figure 1c. At short SOAs, Dell'Acqua 
et al. (2007) observed a nonsignificant -7-ms difference in picture-naming RTs between related and unrelated distractor conditions, and Ayora et al. (2011) observed a difference of $2 \mathrm{~ms}$. However, in the majority of the studies (10 experiments in total, Piai \& Roelofs, 2013; Piai et al., 2014; Schnur \& Martin, 2012; van Maanen et al., 2012), the semantic interference effect was of similar magnitude at shorter and longer SOAs, suggesting that the semantic interference effect emerges during lexical response selection or later stages, as shown in Figure 1b. For example, Schnur and Martin (2012) and Piai et al. (2014) obtained semantic interference effects of 25 to 45 ms regardless of SOA.

Attempts have been made to try to explain the discrepancy between previous findings of additivity and underadditivity observed in the literature (Kleinman, 2013; Piai et al., 2014). Kleinman (2013) proposed that differences in phonological regularity ${ }^{1}$ of the distractor words used in previous studies underlie the observed differences in semantic effects at short SOAs. Whereas phonologically regular words can be read both sublexically by applying grapheme-phoneme correspondence rules as well as via a lexical route, phonologically irregular words do not allow for such a sublexical mapping and are read instead via the lexical route. Kleinman argued that phonologically regular distractor words, such as the ones in Italian used by Dell'Acqua et al. (2007) and Ayora et al. (2011), could be processed concurrently with selecting a response for the tone, before response selection in picture naming. By contrast, for phonologically irregular distractors, such as the ones in English used by Schnur and Martin (2012), the phonological codes generated by the lexical and sublexical routes conflict, thereby prolonging the processing duration of the distractor word beyond response selection for the tone. Assuming a response-selection bottleneck and lexical response-selection locus of semantic interference, as shown in Figure 1b, phonologically regular distractors can already be processed before response selection in picture naming at short SOAs, explaining the underadditive effects of SOA and distractor type of Ayora et al. and Dell'Acqua et al. Phonologically irregular distractors, however, are likely to be processed during response selection in picture naming, explaining the additive effects of SOA and distractor type of Schnur and Martin. However, Piai et al. (2014) used phonologically regular distractor words in Dutch, but obtained additive effects of distractor type and SOA, challenging the phonological regularity account of Kleinman.

According to another account that Kleinman (2013) speculatively considered, the participants of Dell'Acqua et al. (2007) and Ayora et al. (2011) were better readers than those of Schnur and Martin (2012) and Piai et al. (2014). Previous studies have shown that reading skill may be a factor that determines whether additive or underadditive effects are obtained in dual-task performance involving a word recognition task (Ruthruff, Allen, Lien, \& Grabbe, 2008). As readers become more skilled, word processing becomes faster. Therefore, skilled readers may be able to process the distractor word concurrently with selecting a response in Task 1 and before response selection in Task 2, whereas for less skilled readers, distractor processing overlaps with lexical response selection in Task 2. So even if the distractors that participants encounter in the experiment are all phonologically regular (as in Piai et al., 2014), the reading ability of each participant individually may determine the pattern of results. Assuming a response-selection bottleneck and a lexical response-selection locus of semantic interference, as shown in Figure 1b, the semantic interference effect should be reduced or eliminated for skilled readers, but present for less skilled readers at short SOAs.

In the present study, we tested the merits of the reading ability account of the discrepancy between previous findings in the literature. We assessed the reading ability of each participant individually after they performed the PWI task embedded in a

\footnotetext{
${ }^{1}$ Note that phonological regularity is not a property of words per se. Rather, the mapping of graphemes into the corresponding phonemes can be regular or irregular. However, we use the term "phonological regularity" in the remainder of this article to stay close to the proposal by Kleinman (2013).
} 
PRP procedure. Similar to previous studies (for an overview, see Piai et al., 2014), Task 1 was a tone discrimination task with manual responding, and the PWI task (Task 2) had semantically related and unrelated distractors. The SOAs between Tasks 1 and 2 were 0 and 1000 ms. Participants' reading ability was assessed with two different measures, a discrete and a continuous measure, as these are likely to measure their reading ability in different ways. As such, we increased the chances of detecting the influence of reading ability on performance in our PWI task. For the discrete measure, RTs were recorded while participants read aloud each distractor word used in the PWI experiment. For the continuous measure, the One-Minute-Test was used (Brus \& Voeten, 1976), which is a standardized Dutch reading test in which participants read aloud as many words as possible within one minute. Given the discrete nature of picture-naming latencies, we expected the discrete reading measure to better relate to the naming RTs. However, since the One-MinuteTest is a well-established measure of reading ability (also used in clinical settings) in Dutch, we included this measure in our analyses as well. To maximize the need for parallel processing of tone and PWI stimuli (which should yield underadditive effects of distractor type and SOA), the PWI stimuli were presented for only $250 \mathrm{~ms}$, which was shorter than the duration of the tone (300 $\mathrm{ms})$. With short presentation duration, participants have to process the PWI stimuli immediately and cannot postpone their processing until tone processing has been completed. Moreover, the distractor words were phonologically regular, which should also induce parallel processing, according to Kleinman (2013).

The reading ability account predicts a relation between reading times and the size of the semantic interference effect such that the better the reading skill, as indicated by shorter reading times, the smaller the semantic interference effect should be at short SOAs. To test this hypothesis, we conducted linear regression analyses to determine the extent to which reading measures contribute to the magnitude of the semantic interference effect.

\section{EXPERIMENTAL STUDY}

\section{Method}

\section{Participants}

Thirty-five native Dutch speakers (10 male, all university students, mean age $=21.7$ years) participated for course credit or monetary compensation. All participants reported normal hearing and no reading disorders. The data of four participants were discarded due to high error rates (either in Task 1 or 2, or in the order of responding) or great data loss due to instrumental failure.

\section{Materials and design}

The experiment consisted of three consecutive tasks: PRP, discrete reading, and continuous reading. For the PRP task, 32 pictures (eight semantic categories with four objects in each) were used (for materials, see Piai et al., 2014). There were two distractor types. In the related condition, each picture was paired with the name of one of the other pictures from the same semantic category. In the unrelated condition, each picture was paired with the name of one of the pictures from a different semantic category. Thus, in line with the experiments of Piai et al. (2014), all distractor words were part of the response set. Two pure tones (300 and $800 \mathrm{~Hz}, 300 \mathrm{~ms}$ duration) and two SOAs (0 and $1000 \mathrm{~ms}$ ) were used, to make the design more comparable to that in our previous studies (Piai \& Roelofs, 2013; Piai et al., 2014, Experiment 4). Each picture-word stimulus occurred once with each tone at each SOA, with a total of 256 trials, pseudorandomized for each participant individually using Mix (van Casteren \& Davis, 2006). The randomization was constrained such that a given SOA or tone did not appear on more than three consecutive trials. For the discrete reading task, the 32 distractor words (which were also picture names) were used. Each participant received a unique randomized list in which every word occurred twice. For the continuous reading task, the One-Minute-Test was used (Brus \& Voeten, 1976), which is a standardized Dutch reading test containing 116 Dutch words with increasing difficulty. This test is known to 
have a high test-retest reliability of about .87 (Brus \& Voeten, 1976).

\section{Procedure and apparatus}

For the PRP and discrete reading tasks, stimulus presentation and response recording were controlled by the Presentation Software (Neurobehavioral Systems, Albany, CA).

Participants began with the PRP task. Tones were presented to participants via closed headphones, and vocal responses were measured with a voice key. Button presses were measured with a silent button box. Participants were instructed to discriminate the low and high tones via a button press and to name the picture and to ignore the distractor words. Moreover, they were instructed to first respond to the tone and then to the picture-word stimulus. Both speed and accuracy were emphasized. Participants were acquainted with the pictures and their names using a booklet. The experiment started with a practice session. First, participants were familiarized with the tones, and then they performed eight trials of the PRP task. Then the experiment proper began. For the PRP task, at the $0-\mathrm{ms} \mathrm{SOA}$, a trial began with the simultaneous presentation of the picture-word stimulus and the tone. At the 1000-ms SOA, the tone was presented followed by the picture-word stimulus after $1000 \mathrm{~ms}$. The picture-word stimulus always remained on the screen for $250 \mathrm{~ms}$, followed by a black screen for $2750 \mathrm{~ms}$. The recording of the vocal responses started with the presentation of the picture-word stimulus and lasted $3000 \mathrm{~ms}$. After completing the PRP task, the discrete reading task was performed. Participants were instructed to read the distractor words aloud. The words were presented for $1000 \mathrm{~ms}$, followed by a black screen for 500 ms. Finally, for the continuous reading task, participants were instructed to correctly read aloud as many words as possible within the time span of one minute, measured with a stopwatch. They were then given a paper sheet containing the words. Between the task blocks, there was a brief pause. An experimental session lasted approximately half an hour.

\section{Analysis}

The experimenter monitored naming responses online. The following trials were excluded from all analyses: Trials with instrument failure (voice key or button box), trials in which a vocal response was given before the manual response, trials with RTs shorter than $200 \mathrm{~ms}$, and trials with disfluent or incorrect responses. Errors were not analysed due to their low occurrence. For the PRP tasks, by-participant $\left(F_{1}\right)$ repeated measures analysis of variance (rANOVA) was conducted for both the manual and vocal RTs separately, with distractor type (related and unrelated) and SOA (0 and $1000 \mathrm{~ms}$ ) as within-participant variables. For the naming RTs, an additional by-item $\left(F_{2}\right)$ rANOVA was conducted.

Furthermore, for every participant, the average picture-naming RT and the size of the semantic interference effect (mean RT related - mean RT unrelated) were calculated across SOAs, and for the $0-\mathrm{ms}$ and $1000-\mathrm{ms}$ SOAs separately. For the analysis of the discrete reading task, the average reading time for all words was calculated per participant. Finally, for the continuous reading task, the number of correctly named words was taken as the participant's reading score. In addition, to assess test reliability, we calculated participants' average reading time for odd and even trials separately and compared the averages with Pearson's product correlation. Reliability was also calculated for the naming RTs (collapsed over SOA and distractor type) in the same way. Pearson's product correlations between the discrete reading measure, the continuous reading measure, and overall picture-naming RTs were computed. Given that the relation between the picture-naming RTs and the reading measures could be affected by the reliability of our measurements (i.e., an attenuated correlation), we corrected for attenuation using the reliability values obtained (Spearman, 1904). Moreover, linear regression analyses were conducted to assess how much each reading measure contributed to participants' naming RT and semantic interference effect. For each SOA separately and for performance across SOAs, a simple model was fitted with either the continuous or the discrete reading scores as a predictor. 


\section{Results}

\section{Tone task}

Figure 2 shows the mean RTs for the manual responses to the tones (Task 1) for each SOA and distractor type. Tone discrimination was on average $82 \mathrm{~ms}$ slower at the $0-\mathrm{ms}$ than at the 1000-ms SOA, $F_{1}(1,30)=39.7, p<.001$. The main effect of distractor type was not significant, $F_{1}(1,30)<1$, nor was the interaction significant, $F_{1}(1,30)<1$.

\section{Naming task}

Figure 2 also shows the mean RTs for the vocal responses to the pictures (Task 2) for each SOA and distractor type. Naming responses were on average $206 \mathrm{~ms}$ slower at the $0-\mathrm{ms}$ than at the $1000-\mathrm{ms} \quad \mathrm{SOA} \quad\left[F_{1}(1,30)=177.2, p<.001\right.$; $\left.F_{2}(1,31)=792.0, \quad p<.001\right]$, and $36 \mathrm{~ms}$ on average slower with related than with unrelated picture-word stimuli $\left[F_{1}(1,30)=16.4, p<.001\right.$; $\left.F_{2}(1,31)=16.6, \quad p<.001\right]$. Importantly, the semantic effect was of similar magnitude at both

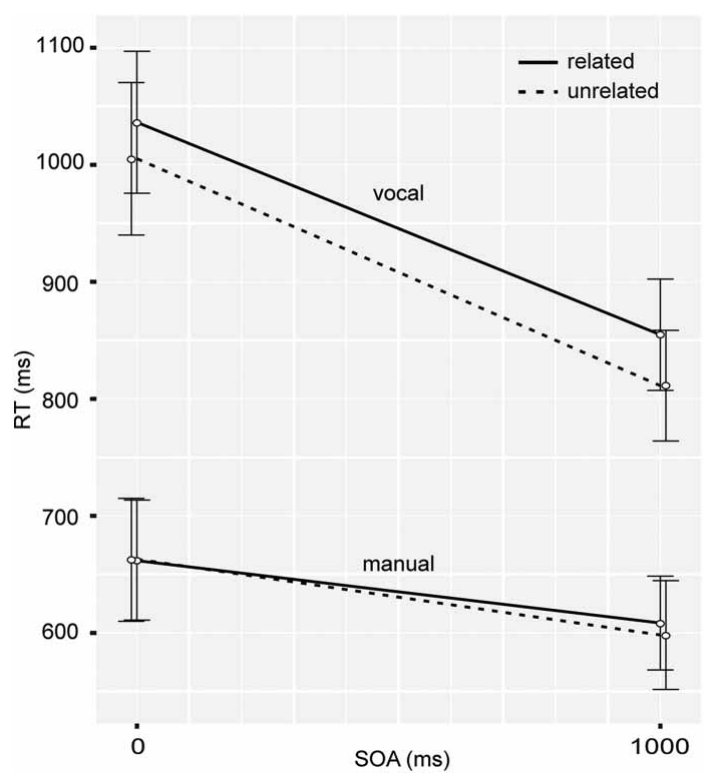

Figure 2. Manual (Task 1) and vocal (Task 2) response times (RTs) as a function of stimulus onset asynchrony (SOA) and distractor type. Error bars indicate 95\% confidence intervals around the mean, calculated from the variance over participants.
SOAs $\left[F_{1}(1,30)<1 ; F_{2}(1,31)=2.3, p=.136\right]$. That is, the effects of distractor type and SOA were additive. The semantic interference effect was on average $31 \mathrm{~ms} \quad(95 \%$ CI $[10,53]$, where $\mathrm{CI}=$ confidence interval) at the $0-\mathrm{ms}$ SOA and $44 \mathrm{~ms}(95 \% \mathrm{CI}[18,69])$ at the $1000-\mathrm{ms}$ SOA.

\section{The role of reading ability}

The continuous reading scores varied between 59 and 116 words and the discrete scores between 350 and $600 \mathrm{~ms}$ (see Figure 3). The continuous and discrete reading scores did not correlate with each other, even after correction for attenuation, $r=-.201, t(29)=-1.106, p=.278$. This indicates that these two tasks measure the participants' reading ability in different ways.

Figure 3 shows the picture-naming RTs and the semantic interference effect as a function of the discrete reading measure at both SOAs, with the regression lines indicated. Table 1 shows the results of the regression analyses for the picturenaming RTs and for the semantic interference effect as a function of the discrete reading measure. Participants' performance in the discrete reading task, as measured by their reading RTs for individual words, was predictive of their naming performance in the PWI task for overall naming and for naming at the $0-\mathrm{ms}$ and $1000-\mathrm{ms}$ SOAs, all $p s<.007$. In contrast, and most importantly, their discrete reading performance was not predictive of the magnitude of the semantic interference effect, all $p s>.639$. Finally, participants' performance in the continuous reading task was not predictive of their naming performance in the PWI task, all $p \mathrm{~s}>.8$, nor of the magnitude of the semantic interference effect, all $p \mathrm{~s}>.7$.

Reliability. The reliability of the discrete reading measure was high, $r=.985, \quad t(29)=31.07$, $p<.001$, as well as the reliability of the picturenaming RTs, $r=.971, t(29)=21.68, p<.001$. Arguably, the fact that discrete, but not continuous, reading times were related to picture-naming times could be explained by the lower reliability for the continuous (.870) than for the discrete (.985) reading task. To test this hypothesis, we examined 

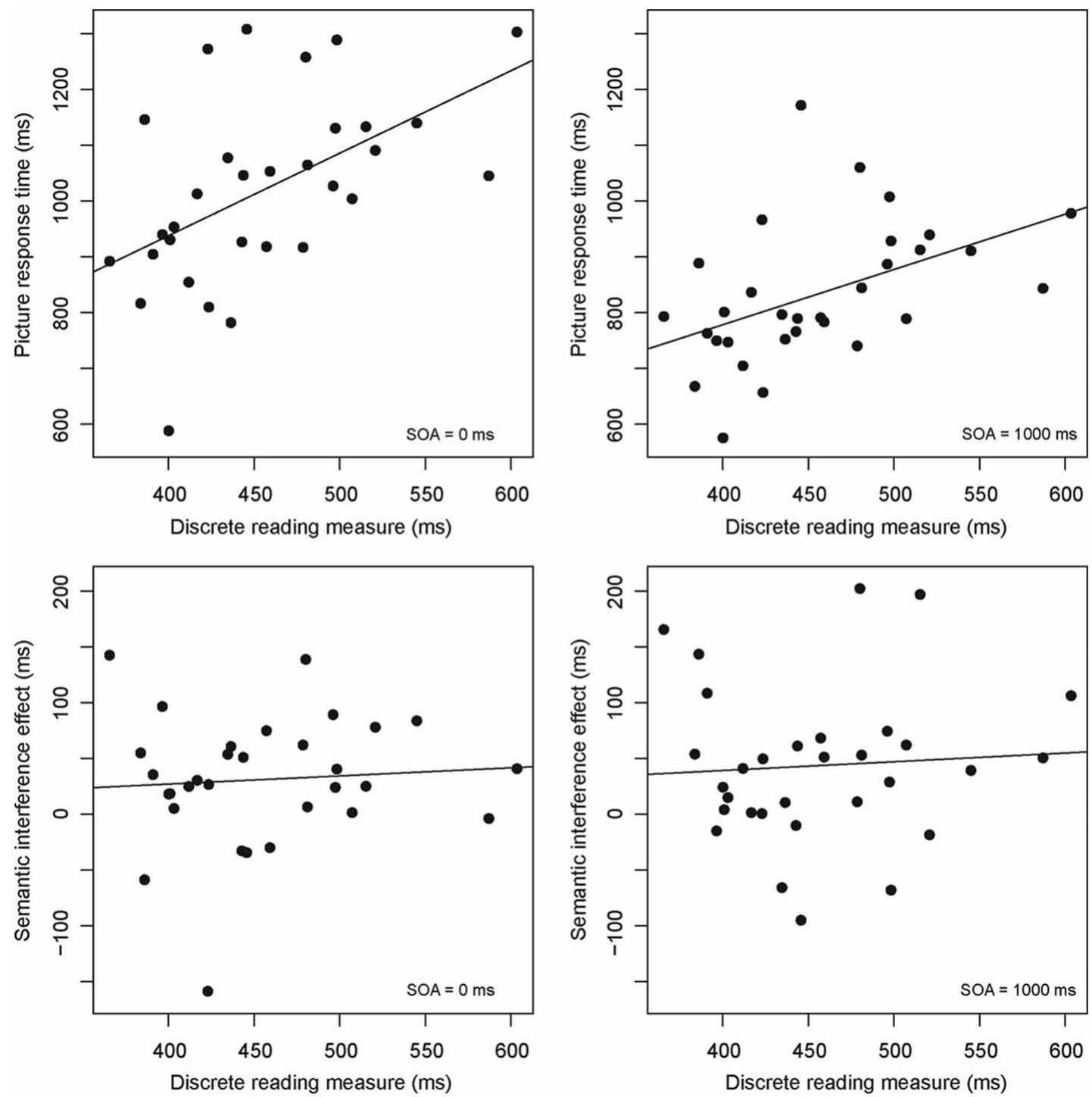

Figure 3. Scatterplots of the relation between the picture-naming response times and the discrete reading measure (upper panels) and between the semantic interference effect and the discrete reading measure (lower panels) at stimulus onset asynchronies (SOAs) of O ms (left panels) and $1000 \mathrm{~ms}$ (right panels). Each point represents one participant. Regression lines are also shown.

the relation between the picture-naming times and the two reading measures while correcting for attenuation. Even after correction, the continuous reading measure still did not have a relation to the naming times, $r=-.038, t(29)<1$, whereas the discrete reading measure still showed a reliable relation to the naming times, $r=.511, t(29)=3.2$, $p=.003$.

\section{Discussion}

In the present study, we examined a possible explanation for the discrepancy observed in the literature regarding additive or underadditive effects of SOA and distractor type in the PRP procedure using PWI and manual tone discrimination. Our participants performed a manual tone task and a 
Table 1. Linear regression models for the naming times and for the semantic interference effect as a function of reading times

\begin{tabular}{|c|c|c|c|c|c|c|c|c|}
\hline Model & & Unstan. $\beta$ & $\mathrm{t}$-value & $\mathrm{p}$-value & $95 \% C I$ & $\operatorname{Adj} . \mathrm{R}^{2}$ & $R S S$ & df \\
\hline \multicolumn{9}{|l|}{ Naming times } \\
\hline \multirow[t]{3}{*}{ Overall naming } & & & & & & .237 & 125.3 & 1,29 \\
\hline & Intercept & 364.21 & 2.06 & .049 & & & & \\
\hline & Reading RT & 1.23 & 3.21 & .003 & $0.45,2.02$ & & & \\
\hline \multirow[t]{3}{*}{ Naming $0 \mathrm{~ms}$} & & & & & & .245 & 147.0 & 1,29 \\
\hline & Intercept & 346.39 & 1.67 & .196 & & & & \\
\hline & Reading RT & 1.48 & 3.28 & .003 & $0.56,2.40$ & & & \\
\hline \multirow[t]{3}{*}{ Naming $1000 \mathrm{~ms}$} & & & & & & .196 & 111.8 & 1,29 \\
\hline & Intercept & 382.04 & 2.42 & .022 & & & & \\
\hline & Reading RT & 0.99 & 2.89 & .007 & $0.29,1.69$ & & & \\
\hline \multicolumn{9}{|c|}{ Semantic interference effect } \\
\hline \multirow[t]{3}{*}{ Mean SIE } & & & & & & -.026 & 52.0 & 1,29 \\
\hline & Intercept & 2.88 & 0.03 & .969 & & & & \\
\hline & Reading RT & 0.08 & 0.47 & .639 & $-0.25,0.40$ & & & \\
\hline \multirow[t]{3}{*}{ SIE $0 \mathrm{~ms}$} & & & & & & -.029 & 59.2 & 1,29 \\
\hline & Intercept & -2.09 & -0.03 & .980 & & & & \\
\hline & Reading RT & 0.07 & 0.40 & .692 & $-0.30,0.44$ & & & \\
\hline \multirow[t]{3}{*}{ SIE $1000 \mathrm{~ms}$} & & & & & & -.030 & 71.0 & 1,29 \\
\hline & Intercept & 7.84 & 0.08 & .938 & & & & \\
\hline & Reading RT & 0.08 & 0.36 & .722 & $-0.37,0.52$ & & & \\
\hline
\end{tabular}

Note: Unstan. = unstandardized; adj. = adjusted; $\mathrm{CI}=$ confidence interval; $\mathrm{RSS}=$ residual sum of squares; $\mathrm{RT}=$ response time; $\mathrm{SIE}=$ semantic interference effect.

PWI task with SOAs of 0 and 1000 ms. The presentation duration of the PWI stimuli was shorter $(250 \mathrm{~ms})$ than the duration of the tones (300 ms), and the distractor words were phonologically regular. After the PRP task, we measured participants' discrete and continuous reading performance.

We observed that the effects of distractor type and SOA were additive (as in Piai \& Roelofs, 2013, and Piai et al., 2014). Our choice of SOA values (0 and $1000 \mathrm{~ms}$ ) was motivated by our attempt to make the present design, and consequently the present results, as similar as possible to those reported by Piai et al. (2014). Therefore, our 0-ms SOA diverted from the more commonly used value of $100 \mathrm{~ms}$ (e.g., Ayora et al., 2011; Dell'Acqua et al., 2007; Kleinman, 2013; Schnur \& Martin, 2012; van Maanen et al., 2012). Note, however, that Piai et al. (2014) also reported an experiment with the 100-ms SOA (Experiment 6) with results highly comparable to those using the 0 -ms SOA. Thus, we have no reason to assume that the present PRP results (with $0-\mathrm{ms}$ SOA) are not comparable to those obtained with the 100-ms SOA.

According to a reading ability account (Kleinman, 2013), reading skill determines whether participants can process the distractor word concurrently with selecting a response to the tone (Task 1) and before response selection in picture naming (Task 2). Processing the distractor word before selecting a response for the picture would decrease the semantic interference effect at short SOAs. Thus, the account predicts that the faster participants can read the distractors, the smaller the semantic interference effect should be. This prediction was not borne out by our data: Participants' reading ability, as measured either discretely or continuously, was not predictive of the magnitude of their semantic interference effect, whereas discrete reading ability was predictive of their picture-naming RT.

The discrete, but not the continuous, reading measure was related to the picture-naming times. 
This finding could not be explained by the lower reliability for the continuous than for the discrete reading task, as assessed by our correlation analysis corrected for attenuation. However, this finding can be explained by taking into account the discrete versus continuous nature of our tasks. Picturenaming performance is measured discretely, rather than continuously. Furthermore, the continuous reading task provides an aggregate measure of reading ability, whereas the discrete reading task measures individual reading responses to words corresponding to the picture names and distractors in the PWI task.

The relation that we observed between discrete reading times and picture-naming times is well in line with the literature showing a relation between people's naming and reading performance (e.g., Denckla, \& Rudel, 1974; van den Bos, Zijlstra, \& lutje Spelberg, 2002; Wimmer, Mayringer, \& Landerl, 2000). Our discrete reading task was powerful enough to detect this often-observed relation between reading and picture-naming speed. To the extent that our discrete reading task provided a measure of participants' reading ability, no evidence was found for the conjecture that reading skill can explain the differences between studies in the literature observing additive or underadditive effects of SOA and distractor type (see Piai et al., 2014, for an extensive discussion of a possible explanation for the differences between studies in terms of a strategic bottleneck).

The present findings on the influence of reading ability in PWI are interestingly different from findings on Stroop task performance. In the Stroop task, participants name the ink colour of incongruent colour words (e.g., the word green in red ink, say "red") or neutral Xs, which yields longer naming RTs in the incongruent than in the neural condition. Protopapas, Archonti, and Skaloumbakas (2007) observed that reading skill influences the magnitude of the Stroop interference effect: The better the reading skill, the smaller the interference. Moreover, Protopapas, Vlahou, Moirou, and Ziaka (2014) observed that word reading practice reduces Stroop interference. The magnitude of the interference is smaller after a few hundred trials of reading aloud the colour words than before practice. To explain these findings, Protopapas and colleagues adopted the distractor blocking account proposed by Roelofs (2003) and implemented in the WEAVER ++ model. On this account, the faster the distractor word is processed, the quicker it can be blocked out, and interference is correspondingly reduced. Protopapas et al. (2007) reported the results of WEAVER++ simulations showing that the model can account for the empirically observed linear relationship between reading speed and the magnitude of Stroop interference. Moreover, Roelofs, Piai, and Schriefers (2011) used this blocking account to explain the distractor-frequency effect in PWI, which is the finding that high-frequency distractor words yield less interference than low-frequency words. According to their account, high-frequency distractor words are processed faster and can thus be blocked quicker than low-frequency words, yielding the difference in interference. However, the present findings indicate that the magnitude of semantic interference is not affected by reading speed, which seems to run counter to the blocking account. On closer look, however, there is no discrepancy. The magnitude of Stroop interference is determined by how quickly an incongruent distractor word is perceptually blocked relative to a series of Xs, the latter not involving word processing. Also, the distractor-frequency effect concerns two word conditions that differ in the speed of perceptual processing. In contrast, the semantic interference effect concerns a difference between related and unrelated words, which do not differ in perceptual processing speed. Consequently, related and unrelated words do not differ in blocking latency, unlike high- and low-frequency distractor words (PWI) or incongruent words and Xs (Stroop). To conclude, the present observation that the magnitude of semantic interference does not vary with reading speed is consistent with earlier findings on Stroop interference and the distractor-frequency effect.

It is important to note the difference between Kleinman's (2013) temporal target-distractor separation account of the additive and underadditive patterns of SOA and semantic effects in dual-task 
performance, on the one hand, and the distractorblocking account of interference effects in singletask performance by Roelofs et al. (2011), on the other hand. According to the account of Kleinman, whether additive or underadditive effects of SOA and semantic relatedness are obtained in dual-task performance depends on whether distractor word processing and response selection in picture naming are temporally separated. If distractor processing is fast, either because the words are phonologically regular or reading is highly automatized, then distractor word processing and response selection in picture naming will be temporally separated, and underadditive effects should be obtained. Instead, if distractor processing is slow, either because the words are phonologically irregular or reading is not much automatized, then distractor word processing and response selection in picture naming will temporally overlap, and additive effects of SOA and semantic relatedness should be obtained. By contrast, the distractorblocking account of interference effects advanced by Roelofs et al. is about the effects of condition differences in the speed of distractor word processing in single-task performance (i.e., picture or colour naming) rather than about effects of concurrent processing on the magnitude of semantic and Stroop interference effects in dual-task performance. As indicated above, differences in the speed of distractor word processing in single-task performance do not need to affect the magnitude of semantic interference.

With respect to PRP performance, we observed that Task $1 \mathrm{RT}$ s were longer at the $0-\mathrm{ms}$ than at the 1000-ms SOA, as also observed by Schnur and Martin (2012) and by Piai et al. (2014, Experiments 4-6). Whereas a structural responseselection bottleneck account predicts that Task 1 RTs should be of comparable magnitude at short and long SOAs, a central capacity sharing account can explain SOA effects on Task 1 RTs. According to this latter account, the bottleneck is not structural but rather arises from central capacity sharing (e.g., Tombu \& Jolicoeur, 2003). If all capacity is first allocated to response selection in Task 1 and then to response selection in Task 2, Task 1 RTs are of comparable magnitude at short and long SOAs, similar to the structural responseselection bottleneck account. Conversely, if capacity is divided between Tasks 1 and 2, response selection processes may overlap and share capacity, thereby slowing down Task 1 RTs, as observed in the present study. However, adjudicating between different accounts of PRP performance falls beyond the scope of the present study.

In conclusion, we observed that although reading $\mathrm{RT}$ s predicted picture-naming $\mathrm{RT}$ s, the magnitude of semantic interference in picture naming during dual-task performance did not vary with reading skill. Thus, the reading ability account does not seem to provide a possible explanation for the discrepancy observed in the literature regarding additive or underadditive effects of SOA and distractor type in the PRP procedure using PWI and tone discrimination.

\section{ORCID}

Vitória Piai (D) http://orcid.org/0000-0002-48605952

\section{REFERENCES}

Abdel Rahman, R., \& Melinger, A. (2009). Semantic context effects in language production: A swinging lexical network proposal and a review. Language and Cognitive Processes, 24, 713-734.

Ayora, P., Peressotti, F., Alario, F.-X., Mulatti, C., Pluchino, P., Job, R., \& Dell'Acqua, R. (2011). What phonological facilitation tells about semantic interference: A dual-task study. Frontiers in Psychology, 2(April), 1-10.

van den Bos, K., Zijlstra, B. J. H., \& lutje Spelberg, H. C. (2002). Life-span data on continuous-naming speeds of numbers, letters, colors, and pictured objects, and word-reading speed. Scientific Studies of Reading, 6, 25-49.

Brus, B., \& Voeten, M. (1976). Een-Minuut-Test (EMT). Berkhout: Nijmegen.

Dell'Acqua, R., Job, R., Peressotti, F., \& Pascali, A. (2007). The picture-word interference effect is not a Stroop effect. Psychonomic Bulletin E Review, 14, 717-722. 
Denckla, M. B., \& Rudel, R. (1974). Rapid "automatized" naming of pictured objects, colors, letters and numbers by normal children. Cortex, 10, 186-202.

Glaser, W. R. (1992). Picture naming. Cognition, 42, 61-105.

Janssen, N., Schirm, W., Mahon, B. Z., \& Caramazza, A. (2008). Semantic interference in a delayed naming task: evidence for the response exclusion hypothesis. Journal of Experimental Psychology: Learning, Memory, and Cognition, 34, 249-256.

Kleinman, D. (2013). Resolving semantic interference during word production requires central attention. Journal of Experimental Psychology: Learning, Memory, and Cognition, 39, 1860-1877.

La Heij, W., Dirkx, J., \& Kramer, P. (1990). Categorical interference and associative priming in picture naming. British Journal of Psychology, 81, 511-525.

Levelt, W. J. M., Roelofs, A., \& Meyer, A. S. (1999). A theory of lexical access in speech production. Behavioral and Brain Sciences, 22, 1-75.

Pashler, H. (1994). Dual-task interference in simple tasks: Data and theory. Psychological Bulletin, 116, 220-244.

Piai, V., \& Roelofs, A. (2013). Working memory capacity and dual-task interference in picture naming. Acta Psychologica, 142, 332-342.

Piai, V., Roelofs, A., \& Schriefers, H. (2011). Semantic interference in immediate and delayed naming and reading: Attention and task decisions. Journal of Memory and Language, 64, 404-423.

Piai, V., Roelofs, A., \& Schriefers, H. (2014). Locus of semantic interference in picture naming: Evidence from dual-task performance. Journal of Experimental Psychology: Learning, Memory, and Cognition, 40, 147-165.

Protopapas, A., Archonti, A., \& Skaloumbakas, C. (2007). Reading ability is negatively related to Stroop interference. Cognitive Psychology, 54, 251-282.

Protopapas, A., Vlahou, E. L., Moirou, D., \& Ziaka, L. (2014). Word reading practice reduces Stroop interference in children. Acta Psychologica, 148, 204-208.

Roelofs, A. (1992). A spreading-activation theory of lemma retrieval in speaking. Cognition, 42, 107-142.

Roelofs, A. (2003). Goal-referenced selection of verbal action: Modeling attentional control in the Stroop task. Psychological Review, 110, 88-125.
Roelofs, A. (2007). Attention and gaze control in picture naming, word reading, and word categorizing. Journal of Memory and Language, 57, 232-251.

Roelofs, A., Piai, V., \& Schriefers, H. (2011). Selective attention and distractor frequency in naming performance: Comment on Dhooge and Hartsuiker (2010). Journal of Experimental Psychology: Learning, Memory, and Cognition, 37, 1032-1038.

Rosinski, R. R. (1977). Picture-word interference is semantically based. Child Development, 48, 643647.

Ruthruff, E., Allen, P. A., Lien, M.-C., \& Grabbe, J. (2008). Visual word recognition without central attention: Evidence for greater automaticity with greater reading ability. Psychonomic Bulletin $\mathcal{\sigma}^{\circ}$ Review, 15, 337-343.

Schnur, T. T., \& Martin, R. (2012). Semantic pictureword interference is a postperceptual effect. Psychonomic Bulletin \& Review, 19, 301-308.

Schriefers, H., Meyer, A. S., \& Levelt, W. J. M. (1990). Exploring the time course of lexical access in language production: Picture-word interference studies. Journal of Memory and Language, 29, 86-102.

Spearman, C. (1904). "General intelligence”, objectively determined and measured. The American Journal of Psychology, 15, 201-292.

Starreveld, P. A., \& La Heij, W. (1996). Time-course analysis of semantic and orthographic context effects in picture naming. Journal of Experimental Psychology: Learning, Memory, and Cognition, 22, 896-918.

Tombu, M., \& Jolicœur, P. (2003). A central capacity sharing model of dual-task performance. Journal of Experimental Psychology: Human Perception and Performance, 29, 3-18.

Van Casteren, M., \& Davis, M. H. (2006). Mix, a program for pseudorandomization. Behavior Research Methods, 38, 584-589.

Van Maanen, L., van Rijn, H., \& Taatgen, N. (2012). RACE/A: An architectural account of the interactions between learning, task control, and retrieval dynamics. Cognitive science, 36(1), 62-101.

Wimmer, H., Mayringer, H., \& Landerl, K. (2000). The double-deficit hypothesis and difficulties in learning to read a regular orthography. Journal of Educational Psychology, 92, 668-680. 